\title{
Remote Laboratory Portal for Robotic and Embedded System Experiments
}

\author{
http://dx.doi.org/10.3991/ijoe.v9iS8.3370 \\ R. Sell \\ Tallinn University of Technology \& ITT Group, Tallinn, Estonia
}

\begin{abstract}
Engineering education process is heavily relying on the practical hands-on experimentation. However, today's education is involving more and more e-learning aspects and learners expect to get most of the content and activity available over the Internet. Practical experiments is not trivial to carry out over the Internet, but using novel ICT technologies and integrated solution, it is possible to offer real experimentation over the Internet. This paper describes and presents the remote practical experiment system in robotic and embedded system domain.
\end{abstract}

Index Terms-e-learning, online laboratory embedded system, remote laboratory, robotics.

\section{INTRODUCTION}

A remote lab (or online lab) enables actors (such as students or employees) to carry out experiments over the Internet, which are normally performed in presence studies at educational laboratories. Compared to a normal laboratory there is need of additional equipment for preparing traditional labs for online access. Remote labs can be divided into distance labs and virtual labs. The main difference between them is that in virtual lab, an experimental environment is simulated instead of mediating the real environment. This approach had some advantages but also disadvantages. If the virtual lab is a software service, once set-up, the lab can be used by a lot of student's simultaneously, only affected by computational power of the host computer. It is also more robust than real equipment as student may not destroy the hardware while adjust some settings or failures in programming. The system can be easily duplicated without paying additional costs. Biggest disadvantage of virtual lab, compared with real one is that a virtual lab may never react in all cases equal to real hardware. It is impossible to include all environmental parameters into the virtualization, thus a virtual lab will react sometimes different from a real one. The best solution is a combination of virtual and remote labs to get benefits from both of them. A general approach, followed of the solution described in this paper is to use the virtual devices for basic education to teach basic system thinking and to get familiar with the hardware. In later steps the learners are switching to real hardware. Closer overview is presented in doctoral thesis [1] and paper [2].

Labs, presented in this paper are about remote laboratories in the field of robotics and embedded systems. During the several EU projects, outcomes presented in several publications [3-5], a base platform is developed to host and mediate different types of remote laboratories around the world. The latest development is under the frame of EU Life Long Learning program project NetLab [6] which established three new lab centers in Estonia, Finland and Germany.

\section{StATE-OF-ART}

Remote labs are gained lot of interest in recent elearning activities. Especially in the engineering field it is clear that classical e-learning tools are not enough to offer engineering courses over the distance.

In Portugal, an initiative lab2go is established to offer shared labs for high school students. The pt.lab2go web platform includes 49 different categories of online experiments, included in 13 laboratories from 7 Portuguese high education institutions [7]. Example experiments are Straightness Evaluation, Meteorological experiments, Chemical Processes, Groundwater flow, etc.

The comprehensive overview about current trends and situation is given in paper [8]. The paper is introducing latest developments and trends in remote and virtual technologies and their application in engineering education. Author gives an overview about the potential of utilizing remote engineering in engineering research, education and professional training, focusing on different kinds of technological characteristics.

Even there are several initiatives, one of the main issues with existing solutions is that they are developed for certain course or particular topic in mind and have applied for local target group. The lab portal described in the following chapters is trying to eliminate these shortages and offer universal portal for offering remote lab as well as sharing the resources between universities and other organizations.

\section{STRUCTURE OF THE E-ENVIRONMENT}

As already mentioned above, the engineering needs today practical labs accessible over the Internet. In the following chapters the DistanceLab remote lab system is described and the connections with learning and teaching tools presented.

The e-environment of remote labs - DistanceLab is a part of Robotic Teaching and Learning Concept presented in the paper [2]. The concept offers wide range of tools and methodology to effectively and interactively teach embedded systems and robotics as well as exploit latest web technologies.

The whole environment consists of several logical servers which can physically locate in one server or distributed over the many different locations. The current setup consists of one portal server and every lab location has its own programming server. In addition, all labs and also all devices can have their own real-time audio-video feedback system.

The lab is consisting of number of similar equipment with wired or short range wireless communication mod- 
ule. The site is fitted with web camera and server which communicates with the robots. Server has a master communication unit which can be contact with any robot on the field and reprogram it at any time when needed. The site server is connected with the portal server passing and validating the communication between the robot and user input.

Remote Lab Centre (RLC) is a specific location-based lab which can be accessed over the Internet. A Remote Lab Center focuses on specific equipment or a series of experiments by giving online access, enabling control and monitoring of the process. Remote Lab Centres are located in different organizations like universities, vocational schools, SME-s and offer through a resource sharing concept, different remote labs, such as; mobile robot lab, microcontroller test bench, automotive lab and smart house lab. In principle, RLC can be either equipped with real hardware named as a DistanceLab or offer virtualized hardware simulator - VirtualLab.

\section{DEMONSTRATION OF LABS}

Remote Lab Centres are currently up and running as prototypes in following locations:

- Tallinn and Võru, Estonia

- Helsinki, Finland

- Bochum, Germany

In this chapter following remote labs will be shortly described:

1. Robotic HomeLab test bench in Robolabor (Fig. 1.)

2. Mobile Robot lab in VKHK, Võru (Fig. 2.)

3. Robot manipulator lab in Robolabor and Bochum University of Applied Sciences (Fig. 3.)

4. Smart Greenhouse in VKHK, Võru (Fig. 4.)

\section{A. Robotic HomeLab kit test bench}

Robotic HomeLab test bench is based on Robotic HomeLab kit where the kit is assembled into small test bench system. The system consists of standard components from Robotic HomeLab Basic and Add-on kits. In particular, DC motor, stepper motor and servo motor as actuators and infrared, ultrasonic distance sensors, temperature sensor and photo resistor as sensors. Students can perform several exercises assigned by supervisor over the Internet by using this remote lab. All exercises are published in the Network of Excellence - NoE and can be accessed also remotely. The feedback of programmed controller behaviour can be acquired from online videofeedback system where all test benches have their own personal camera.

\section{B. Mobile Robot Lab}

Mobile robot lab is a set of similar mobile robots driving around the arena. Robots can be booked over the DistanceLab portal by the users with sufficient rights. Robots are based on RP06 tracked robot platform equipped with ultrasonic distance sensor, wheel encoders and line following infrared sensors. The difference between previous lab and mobile robot lab is the camera interface. In Robotic HomeLab test bench, every device has its own personal camera whereas mobile robot lab has only two general overview cameras. As robots are moving around it is impossible to focus camera to one static point as well as user need broader overview what happening on the robot field and how his/her robot behaves.

\section{Manipulator Lab}

In this remote lab, a welding robot is simulated, which is one of the more widespread applications of manipulators in the processing industry, particularly the car industry. The welding robot will be simulated in the learning situation by a simplified manipulator with 5 freedom stages, its links controlled by RC servomotors. The manipulator or robot arm is positioned on a moving base that can be moved along one axis with the help of a direct current motor, to which an encoder is in turn connected, making it possible to obtain feedback about the actual movement of the base. The scope of movement of the base is limited by mechanical limit switches. As welding is an operation that poses a significant fire hazard, its actual application in a remote lab by students is complicated and there is too much risk involved. Therefore, the welding element has been replaced with a usual marker pen that is comfortable and safe to manipulate over the Internet (see Fig. 3.).

\section{Smart Greenhouse}

The aim of a smart house concept is to concentrate all of the functions controlled by the house into one system, offering increased comfortability, security and reduced energy consumption that are achieved via intelligent control algorithms. What makes the house intelligent is the joint impact of these functions and an autonomous regula-

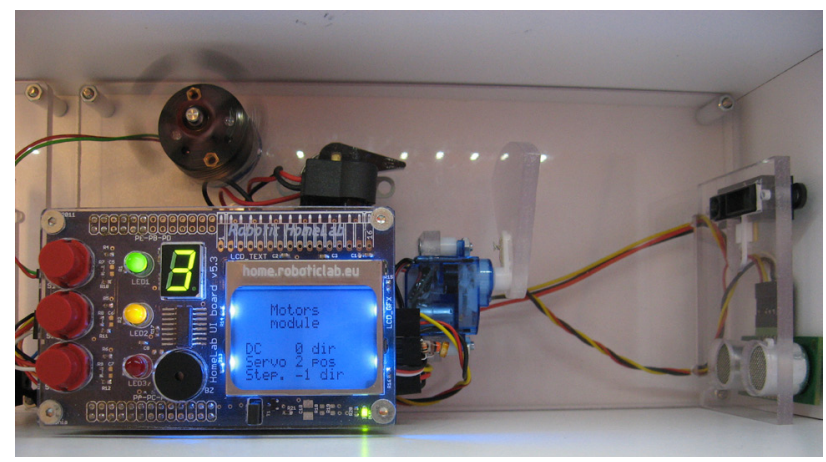

Figure 1. Robotic HomeLab kit test bench in Robolabor

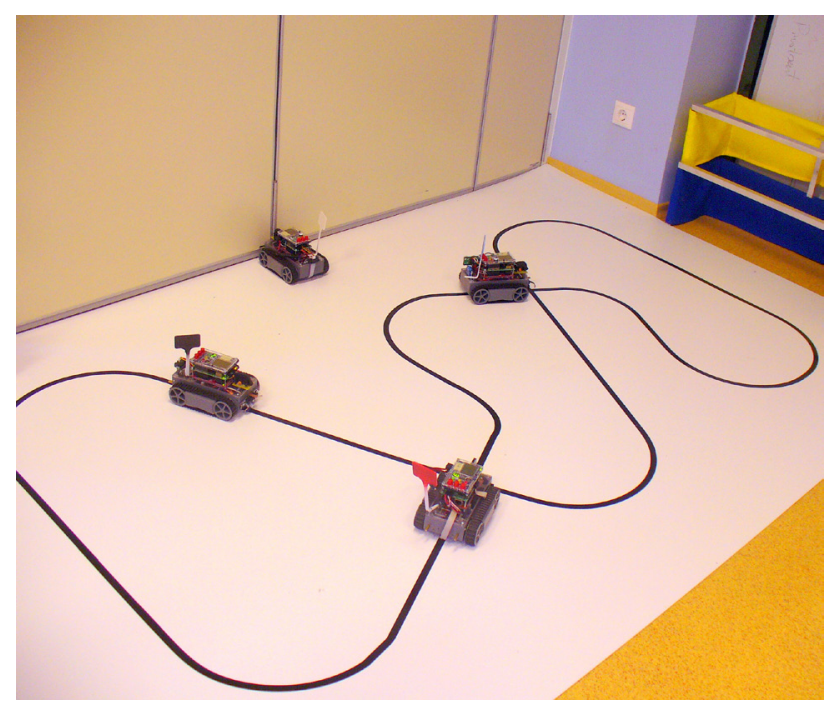

Figure 2. Mobile Robot lab in Võru 


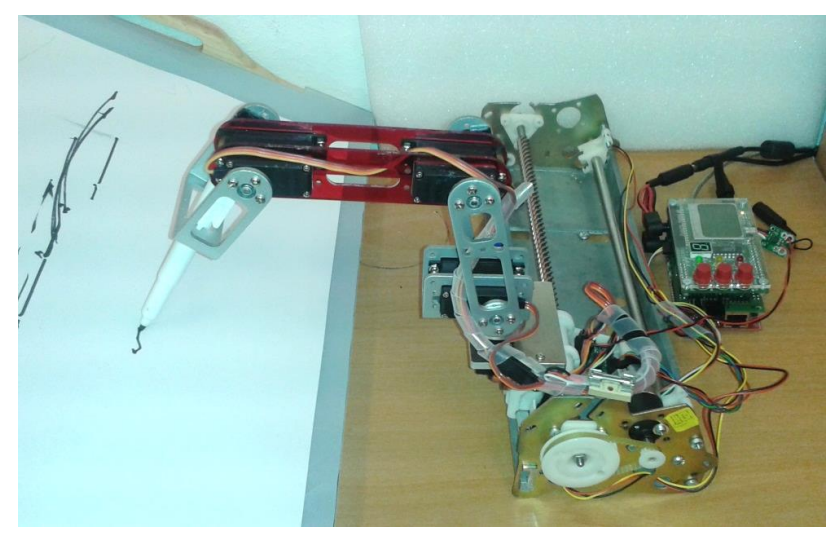

Figure 3. Robotic HomeLab kit test bench in Robolabor

tion feature. An intelligent house creates a secure, economic, comfortable and healthy living environment and has the functionalities of remote monitoring and control. According to the intelligent house concept, maximum comfort should be created for the inhabitants of the house while economizing on resources. A similar concept can be applied to growing plants in a greenhouse, where the smart house concept is transformed into the smart greenhouse concept that aims to optimize growth conditions for plants and automate the growing process. There are a lot of work and activities around a greenhouse, but some of these activities are routine and relatively easy to automate. As day and night temperatures vary considerably in our climate, the greenhouse has to close hatches in the evening and reopen them in the morning, so that the plants would not be too cold in the night and not harmed by the sun in daytime. Without automation, the gardener depends on the greenhouse. Besides air temperature, air and soil moisture also have to be kept under control in the greenhouse. Stormy winds are not uncommon; these can harm the plants or the greenhouse construction. In the case of a storm, all hatches need to be closed. The following recommended activities can be listed for a greenhouse and need to be solved in a systematic manner:

- adjusting inner climate parameters on the basis of the readings of outer and inner climate sensors,

- moisturizing soil on the basis of the readings of soil moisture sensors,

- collecting sensor readings and controlling regulators,

- logging sensor readings into a database and displaying them graphically,

- wireless interconnection of modules,

- using alternative energy (solar and wind energy) for heating the irrigation water and recharging the batteries,

- feeding the plants and adjusting the lighting,

- auxiliary functions related to power supply, computer communication, security.

The remote lab developed for controlling the intelligent greenhouse is established in Voru. The concept behind the educational smart greenhouse is that agriculture and mechatronic students can work together as an interdisciplinary team. Student teams have to develop the growing conditions for tomato and develop the algorithm for reading sensors and controlling actuators. Educational remote greenhouses are developed and included in the DistanceLab portal so that team members from different

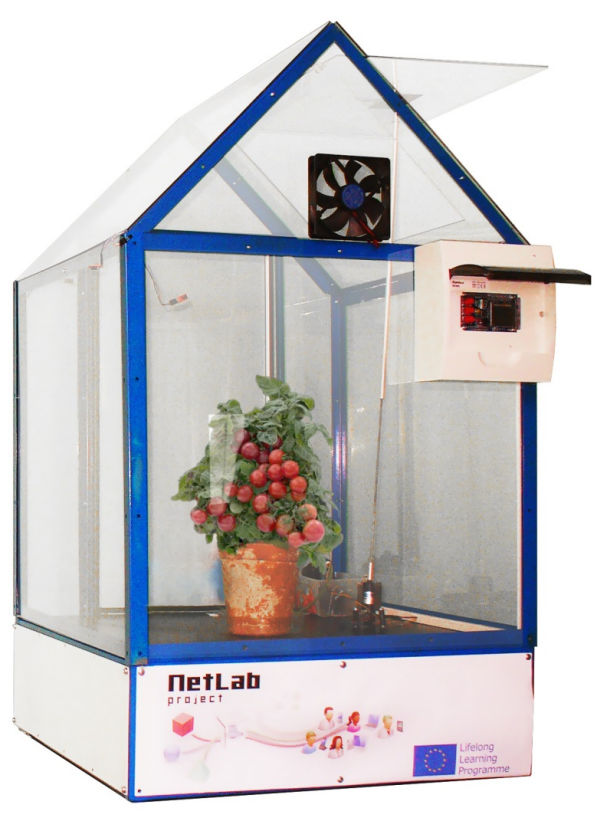

Figure 4. Smart Greenhouse remote lab hardware

schools in different location can control and monitor their own greenhouse at any place in any time. The remote lab educational greenhouse is seen in Fig. 4.

All previously described remote labs are located in Estonia, but different organizations and different cities. Labs are actively used by vocational schools and gymnasium teachers to practice their knowledge in embedded system programming. Some of the same types of labs are also available in Germany and Finland. Mentioned labs and learning situation which can be performed on these labs are published in the book [9].

\section{Distancelab Portal}

The remote lab environment portal offers a complete remote lab management and programming environment for remote labs - either distance labs or virtual labs. The functionality connected with remote lab is as following:

- user and group management,

- location, lab and device management,

- source code validation and version management,

- wireless device communication,

- device booking and booking rights.

Having a broad view in mind, the system supports three levels of grouping:

- Location - this is organizational level where different type of devices and labs can exist,

- Lab - the virtual room where physically or virtually different devices can be located,

- Device - a set of same type of devices in one location.

Devices have additionally grouped into several subgroups according their type, e.g. mobile robots, manipulators, PLC-s, etc. Video feedback system can be connected to one lab (usually two cameras) or to every device. The number and focus of video cameras is related to the nature of the lab. In case of moving objects like mobile robots, usually lab has general cameras focusing on the arena where robots can drive around. In case of attached device 
like Robotic HomeLab test bench or manipulator, every device has its own camera. In this case camera can precisely focus to the device.

The remote lab management website http://distance. roboticlab.eu, is an interface to access different remote labs (e.g. labs demonstrated before). Fig. 5. presents the view of one particular lab where use can see the description of the lab, the physical location on Google map, two real-time video output and list of the devices offered by the lab.

The Fig. 6. shows the programming interface of one particular lab. In this case a C-language programming interface is implemented for controlling the Robotic HomeLab kit test bench remote lab.

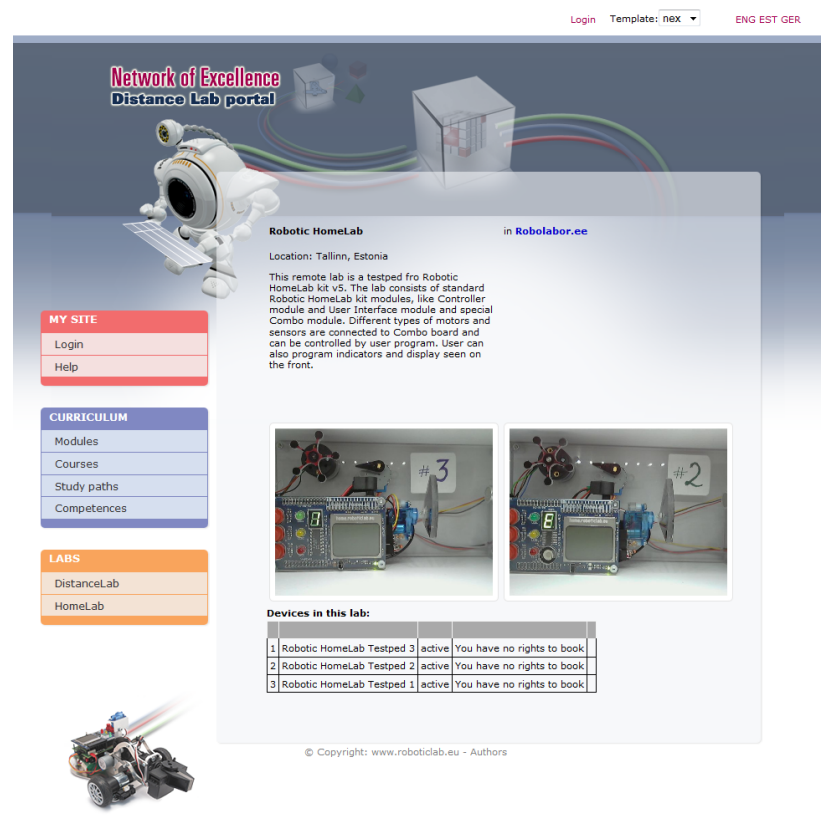

Figure 5. Lab overview in DistanceLab portal

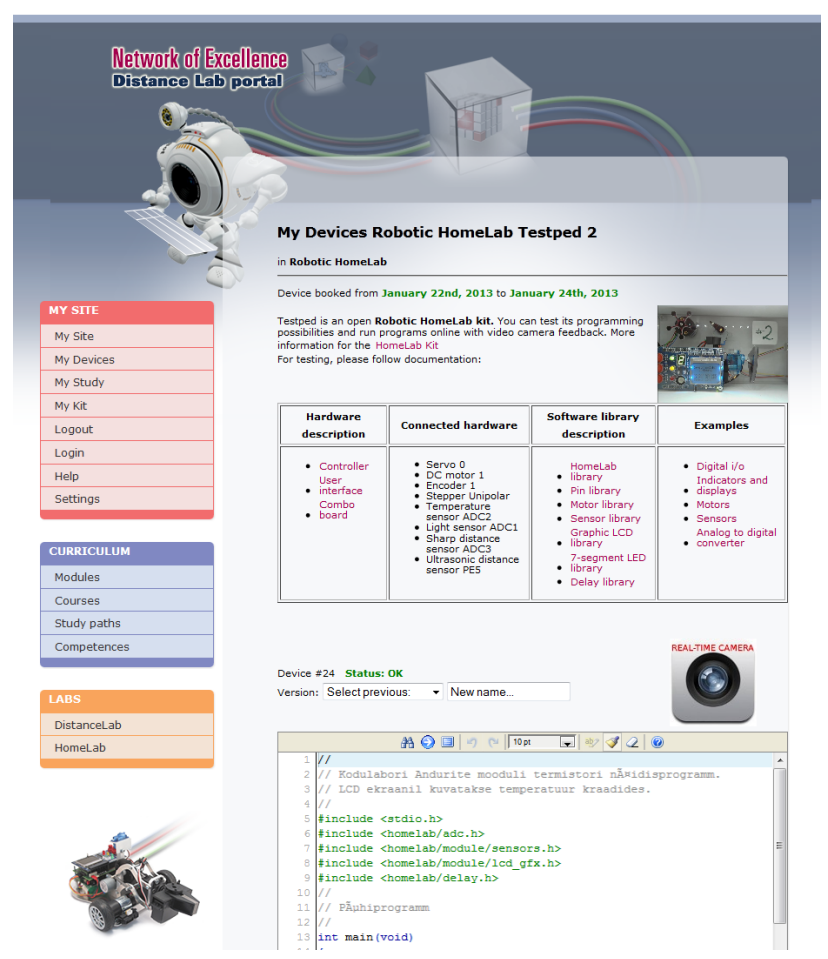

Figure 6. Programming interface in DistanceLab portal

\section{CONCLUSIONS}

In summary, the overview of remote lab system DistanceLab is given and main functionality is explained. Four different types of lab, namely: Robotic HomeLab kit test bench, Mobile Robot lab, Manipulator lab and Smart Greenhouse lab is described in more detail. All mentioned labs are up and running by offering the remote access and engineering experiments over the web. Even not all labs are available for public some of them can be accessed by asking the access from lab owner. Several labs described in the paper are developed on the frame of Life Long Learning program project, NetLab [8]. The concept is applied into practice for different target groups in Estonia, Germany and Finland. The feedback from students is very positive and they truly appreciate new technology enhanced learning as well as project based learning through the learning situations. New component development of learning system is in progress and new target groups and sectors will be involved in near future.

\section{REFERENCES}

[1] S. Seiler, supervisors R. Sell, R. Laaneots. Laboratory as a Service - A Holistic Framework for Remote and Virtual Labs. Ph.D thesis, Tallinn University of Technology Press, 2012.

[2] R. Sell and S. Seiler, Learning Situations and Remote Labs in Embedded System Education, in REM2013, Vienna, 2013. "in press"

[3] S. Seiler, R. Sell, D. Ptasik and M. Bölter, Holistic web-based Virtual Micro Controller Framework for research and education, in International Journal of Online Engineering, vol 8, nr 4, pp. 5864, 2012.

[4] R. Sell, S. Seiler, Improvements of Multi-disciplinary Engineering Study by Exploiting Design-centric Approach, Supported by Remote and Virtual Labs, in International Journal of Engineering Education, vol. 28, issue 4, pp. 759-766, 2012.

[5] S. Seiler, R. Sell and D. Ptasik, Embedded System and Robotic Education in a Blended Learning Environment Utilizing Remote and Virtual Labs in the Cloud, Accompanied by 'Robotic HomeLab Kit', International Journal of Emerging Technologies in Learning, Vol 7, Issue: 4, pp. 26-33, 2012.

[6] Learning Situations for Embedded System Study Lab - NetLab, 2011-0019-LEO05-TOI-01, Life Long Learning project, 2010.

[7] M.T. Restivo and A. Cardoso, The Portuguese Contribution for lab2go - pt.lab2go, in International Journal of Online Engineering, vol. 9, Special Issue 1: "REV2012 Exhibition", 2013

[8] S. Seiler, Current Trends in Remote and Virtual Lab Engineering. Where are we in 2013?, in International Journal of Online Engineering, vol. 9, issue 6, 2013

[9] Learning Situation in an Embedded Systems, Ed. R. Sell, Robolabor Publisher, Tallinn, 2013

\section{AUTHORS}

R. Sell is with the Tallinn University of Technology senior researcher and R\&D manager ITT Group, Estonia (e-mail: raivosell@gmail.com).

This work was supported in part by EU Life Long Learning program projects 2011-0019-LEO05-TOI-01 "Learning Situations in Embedded System: StudyLab". The further transfer of innovation is supported by LLL projects USORA and ViReal. Research part was supported by ETF grant 8532. This article is an extended and modified version of a paper presented at the International Conference exp.at'13, held 18-20 September 2013, in Coimbra, Portugal. Submitted 18 November 2013. Published as re-submitted by the authors 04 December 2013. 